ESJ Humanities

\title{
La démocratie ivoirienne à l'épreuve des stratégies coalitionnelles politiques
}

\author{
Dr. Diarassouba Ibrahima, Sociologue
}

Université Alassane Ouattara de Bouaké, Côte d'Ivoire

Doi:10.19044/esj.2021.v17n28p150

Submitted: 19 August 2020

Accepted: 16 July 2021

Published: 31 August 2021
Copyright 2021 Author(s)

Under Creative Commons BY-NC-ND

4.0 OPEN ACCESS

Cite As:

Ibrahima D. (2021). La démocratie ivoirienne à l'épreuve des stratégies coalitionnelles politiques. European Scientific Journal, ESJ, 17 (28), 150.

https://doi.org/10.19044/esj.2021.v17n28p150

\section{Résumé}

L'adoption du multipartisme par la Côte d'Ivoire en 1990 s'est accompagnée du phénomène de la coalition comme nouvelle stratégie de conquête du pouvoir par des partis politiques à travers des alliances politiques. La première coalition (le front républicain) visant à former une majorité électorale est née en 1995 de l'alliance entre le FPI et le RDR pour évincer le PDCI du pouvoir. Ensuite s'en sont suivi le Rassemblement des Houphouétistes pour la Démocratie et la Paix (RHDP) en 2005, et La Majorité Présidentielle (LMP) en 2010. Ces coalitions ont toutes fait preuve de fragilité face aux opportunités politiques au point que deux d'entre elles n'existent plus (Front Républicain ; LMP), et celle qui existe (RHDP), est exposée au risque de désintégration à cause des querelles internes de positionnement. Le présent article a pour objectif d'identifier et d'analyser des causes des échecs des coalitions politiques et leurs conséquences sur la démocratie en Côte d'Ivoire. C'est une étude qualitative ancrée dans le courant théorique du changement social. Elle s'appuie sur la méthode de l'analyse de contenu. Après l'analyse des données, quatre principales causes de la fragilité des coalitions ont-été identifiées. Il s'agit : (1) du charisme trop fort des leaders politiques dans la coalition ; (2) du non-respect du principe d'inclusivité dans la prise de décision par les partis dans la mise en place des coalitions (3) de l'inexistence d'un projet de société commun clairement formalisé dès le départ entre les partis coalisés; (4) du comportement nombriliste des partis politiques membres des coalitions qui nourrissent des projets personnels inavoués.

Mots clés : Acteurs, Coalition Politique, Reconfiguration politique 


\title{
Ivorian Democracy put to the Test of Political Coalition Strategies
}

\author{
Dr. Diarassouba Ibrahima, Sociologue \\ Université Alassane Ouattara de Bouaké, Côte d'Ivoire
}

\begin{abstract}
The adoption of multiparty politics by the Ivory Coast in 1990 was accompanied by the phenomenon of the coalition as a new strategy for the conquest of power by political parties through political alliances. The first coalition (the Republican Front) aimed at forming an electoral majority arose in 1995 from the alliance between the FPI and the RDR to oust the PDCI from power. Then followed the Gathering of Houphouetists for Democracy and Peace (RHDP) in 2005, and The Presidential Majority (LMP) in 2010. These coalitions have all shown fragility in the face of political opportunities to the point that two of them between them no longer exist (Republican Front; LMP), and that which exists (RHDP) is exposed to the risk of disintegration because of internal disputes over positioning. The objective of this article is to identify and analyze the causes of the fragility of political coalitions and their consequences on democracy in Côte d'Ivoire. It is a qualitative study rooted in the theoretical stream of social change. It is based on the method of content analysis. After analyzing the data, four main causes of the fragility of coalitions were identified. These are: (1) the too strong charisma of political leaders in the coalition; (2) the failure to respect the principle of inclusiveness in decision-making by the parties in the establishment of coalitions (3) the non-existence of a common social project clearly formalized from the start between the united parties; (4) the navel-gazing behavior of the political parties that are members of the coalition, which have unacknowledged personal projects.
\end{abstract}

Keywords: Political coalition, Political reconfiguration, Actors

\section{Introduction}

L'histoire sociopolitique de la Côte d'Ivoire multipartite rime à la fois avec la radicalisation de son arène politique avec son corolaire d'instabilité sociopolitique continue, et le phénomène des coalitions politiques comme nouvelle stratégie des partis politiques pour réaliser l'alternance au pouvoir. En effet, l'instauration du multipartisme qui marque la naissance de la démocratie (toujours en construction) en côte d'ivoire, s'est accompagnée de remous sociaux qui ont jusque-là plongé la Côte d'Ivoire dans une instabilité politique. Durant la décennie 1990 à 1999, face à un régime au pouvoir (PDCI) 
très oppressif, se trouve une opposition politique radicale supplantée par Front Populaire Ivoirien (FPI) et le Rassemblement Des Républicains (RDR. Dans cette atmosphère de torpeur et d'incertitude pour les ivoiriens, l'armée intervient à travers un coup d'Etat le 24 décembre 1999 pour «balayer la maison et y mettre de l'ordre et sauver le pays d'un chaos ${ }^{1}$. Ce coup d'Etat, loin d'avoir été salvateur, semble avoir été le point de départ de brutalisation du champ politique ivoirien, avec successivement le charnier de Yopougon à la suite des élections « calamiteuses $»^{2}$ de 2000, suivi de la rébellion armée de 2002 dont on ignore encore le nombre exact de victimes, et la crise postélectorale de 2010 ayant officiellement fait 3000 morts.

Tous ces évènements ont eu pour conséquences (1) la faillite de l'Etat matérialisé désormais par son incapacité à exercer sur les espaces publics son autorité contre les violences (Babo, 2008, ); (2) l'engagement dans les violences politiques des jeunes gagnés par le chômage qui apparaît comme étant l'aboutissement normal d'un long processus de maturation porté par une construction lente dans la violence (Babo, 2011).

Sur le plan démocratique, on assiste à des processus électoraux non inclusifs, des violences électorales, l'absence d'alternance pacifique, l'inexistence d'un Etat de droit, la restriction de l'espace civique et la violation des droits civils et politiques. C'est dans ce contexte qu'ont été mises en place plusieurs coalitions politiques à la recherche de la majorité électorale pour conquérir le pouvoir.

La première coalition politique a commencé en 1995 par le Front Républicain à travers une alliance entre le Front Populaire Ivoirien (FPI) et le Rassemblement Des Républicains (RDR). D'autres coalitions ont suivi le Front Républicain. Il s'agit du Rassemblement des Houphouétistes pour la Démocratie et la Paix (RHDP) en 2005 et de La Majorité Présidentielle (LMP) en octobre $2010^{3}$ (Jeune Afrique, 2010). Toutefois, ces coalitions ont toutes fait preuve de leur irrésistibilité au temps face aux opportunités politiques.

Le Front Républicain s'est dissout en 2000 après le coup d'Etat du 24 décembre 1999. La LMP a disparu d'elle-même après l'arrestation en avril 2011 de l'ex-président de la république Laurent Gbagbo suivi de son transfèrement à la Cour Pénale Internationale (CPI). En 2017, apparaissent les signes de la crise au sein du RHDP avec la question de l'alternance au pouvoir en 2020 entre le RDR et le PDCI. L'accentuation de cette crise a suscité le

\footnotetext{
${ }^{1}$ Affirmation de Feu, général Robert Guéi, qui a assuré la transition pour justifier le coup d'Etat

${ }^{2}$ Le président Laurent à la suite de son élection en affirme qu'il a été élu dans des conditions calamiteuse

${ }^{3}$ Jeune afrique du 10octobre 2010
} 
départ de plusieurs partis membres dont le PDCI, le MFA, l'UDPCI, l'UPCI, le PIT $^{4}$.

Au regard de ces constats, il se pose donc la problématique de la capacité des coalitions politiques à se maintenir durablement et réaliser le changement politique en Côte d'Ivoire. Cela nous amène à nous questionner sur les causes de leurs échecs et leurs conséquences sur la démocratie en Côte d'Ivoire. Notre article tente d'apporter une réponse à cette interrogation à partir d'une analyse du cadre coalitionnel dans l'arène politique en Côte d'Ivoire. Pour mieux rendre compte, il s'articule autour de trois grands points :

I- La méthode d'analyse et la technique de collecte des données;

II- L'approche conceptuelle et théorique

III- L'analyse du cadre coalitionnel politique en Côte d'Ivoire

\section{La méthode d'analyse et la technique de collecte des données I.1. La technique de la collecte des données}

Dans la réalisation de l'étude, outre les entretiens réalisés à partir de guide d'entretien avec les militants de certains partis politiques (RDR, PDCI, FPI), nous avons privilégié les discours des leaders politiques sur les réseaux sociaux. En effet, s'il y a une réalité qui, indéniablement a fortement porté un chamboulement dans la stratégie de communication du monde politique, c'est bien l'existence des réseaux sociaux et leur facilité d'accès aux militants des partis politiques notamment les jeunes ${ }^{5}$.

Selon les données publiées par l'Institut National de la Statistique (INS, 2014) relatives au recensement général de l'habitat et de la population de 2014, la population ivoirienne est majoritairement en référence à la tranche d'âge de 0 à 15 ans $^{6}$ (cf-tableau ci-dessous). Cette jeunesse influe certainement sur l'électorat des partis politiques.

La Côte d'Ivoire n'échappe à cette réalité et son arène politique n'est pas exempte de ce chamboulement favorisé par ces médias sociaux devenus désormais des lieux d'exercice d'âpreté du combat politique, d'invectives par la virulence des discours ${ }^{7}$. Ainsi, l'opposition politique tant à se mener plus sur ces nouveaux canaux de communication. Les verbatim extraits des discours des leaders politiques dans cet article ont été pris sur Youtube.

\footnotetext{
4 Tous ces partis connaissent deux tendances. Une tendance dirigée par leurs premiers responsables qui est membre de l'opposition, et l'autre tendance gérée par des cadres desdits partis et est membre du RHDP

${ }^{5}$ De lus en plus courtisés par les partis politiques pour accroitre leur électorat.

${ }^{6} \mathrm{La}$ jeunesse selon les normes internationales universellement admises notamment celles des Nations unies, se situe entre 18 et 35 ans.

${ }^{7}$ D'après un cadre du RHDP « Les réseaux sociaux sont devenus les lieux d'injures pour les leaders politique et leurs des militants»
} 
Tableau 1: recensement général de l'habitat et de la population de 2014

\begin{tabular}{|l|l|l|}
\hline $\begin{array}{l}\text { Répartition } \\
\text { par zone }\end{array}$ & Effectif & $\begin{array}{l}\text { Taux } \\
(\mathbf{\%})\end{array}$ \\
\hline $\begin{array}{l}\text { Population } \\
\text { totale }\end{array}$ & 22.671 .331 & $\mathbf{1 0 0 , 0}$ \\
\hline $\begin{array}{l}\text { Population } \\
\text { en zone de } \\
\text { forêt }\end{array}$ & 17.107 .086 & $\mathbf{7 5 , 5}$ \\
\hline $\begin{array}{l}\text { Population } \\
\text { en zone de } \\
\text { savane }\end{array}$ & 5.564 .245 & $\mathbf{2 4 , 5}$ \\
\hline $\begin{array}{l}\text { Population } \\
\text { rurale }\end{array}$ & 11.262 .918 & $\mathbf{4 9 , 7}$ \\
\hline $\begin{array}{l}\text { Population } \\
\text { urbaine }\end{array}$ & 11.408 .413 & $\mathbf{5 0 , 3}$ \\
\hline
\end{tabular}

\begin{tabular}{|l|l|l|}
\hline $\begin{array}{l}\text { Caractéristiques } \\
\text { par Sexe et par âge }\end{array}$ & Effectif & $\begin{array}{l}\text { Taux } \\
(\mathbf{\%})\end{array}$ \\
\hline Homme & 11.708 .244 & $\mathbf{5 1 , 7}$ \\
\hline Femme & 10.963 .087 & $\mathbf{4 8 , 3}$ \\
\hline 0 à 15 ans & 9.481 .351 & $\mathbf{4 1 , 8}$ \\
\hline 15 à 65 ans & 12.609 .533 & $\mathbf{5 5 , 7}$ \\
\hline Plus de 65 ans & & $\mathbf{2 , 5}$ \\
\hline
\end{tabular}

Source : INS, 2015

\section{I.2. L'analyse de contenu}

L'analyse de contenu permet d'extraire les contenus signifiés d'un discours, qu'il soit écrit, oral, etc. Car, tout document écrit, parlé ou sensoriel contient potentiellement une quantité d'informations sur la personne qui en est l'auteur, sur le groupe auquel il appartient, sur les faits et évènements qui y sont rattachés, sur les effets recherchés par la présentation de l'information, sur le secteur réel dont il est question (Dantier, 2008- Mucchielli, 2006). Dans le cadre d'une étude, elle consiste à partir d'une analyse d'extraire le contenu signifié contextualisé du discours. Dans cet article, nous avons procédé à l'analyse d'extraits des discours de certains leaders politiques ${ }^{8}$ que nous trouvons très significatifs en lien avec le changement politique recherché.

\section{Approche conceptuelle et théorique \\ II.1. Approche conceptuelle}

La coalition et l'alliance sont des notions plus présentes dans le vocabulaire politique qu'il convient dans le cadre de notre étude de conceptualiser en vue d'une meilleure compréhension. Il s'agit pour nous à travers une analyse de savoir, du point de vue sémantique et empirique s'ils sont synonymes et désignent la même réalité ou, si chacune d'elles désigne une réalité particulière.

(Duverger, 2003) et Haeringer (2003) font un usage alterné des notions de coalition et alliance pour désigner un même fait politique, qui consiste pour certains partis politiques décider de se mettre ensemble et constituer la

${ }^{8}$ Le président de la république, Henri Konan Bédié président du PDCI-RDA, Pascal Affi N'guessan président du FPI, Ibrahima Cissé Bacongo membre du bureau politique du RDR, Konan Kouadio Bertin et Zié Daouda Coulibaly tous deux membres du bureau politique du PDCI-RDA 
majorité électorale. Toutefois, à l'intérieur de ces coalitions naissent naturellement des rivalités entre les partis coalisés, faisant que cette majorité devienne moins cohérente, moins homogène, et moins stable (Duverger, op.cit.). Allant dans le même sens, Haeringer (op.cit), dans son analyse des coalitions en Afrique, relève l'absence d'une coopération totale entre les partis membres. Ces coalitions fonctionnent sur la base d'une «coopération partielle », en faisant le choix de coopérer seulement dans certains types d'action à l'aune des enjeux politiques du moment et des opportunités qui se présentent pour chacun des membres coalisés.

C'est avec Michelle et Karine à travers la typologie des regroupements politiques qu'ils établissent qu'apparait une différence entre l'alliance et la coalition liée à la temporalité et le niveau d'organisation. En fonction du régime politique et des enjeux électoraux, on peut avoir une coalition ou alliance électorale, une coalition ou alliance parlementaire, une coalition ou alliance gouvernementale. Ils utilisent la notion de coalition pour désigner les formes de regroupement politique éphémère et inorganisé dans le but de créer une majorité électorale, qu'ils qualifient de simples coalitions provisoires dont les partis membres ne cherchent qu'à bénéficier des avantages électoraux, soit en soutenant occasionnellement le parti au pouvoir, soit en contribuant à l'évincer du pouvoir. Ils utilisent la notion d'alliance pour désigner les unions politiques durables (Michelle et Karine, 2017) ${ }^{9}$.

Toutes ces analyses mettent en exergue un invariant du phénomène coalitionnel ou d'alliance. Il s'agit de sa présence plus remarquée dans les systèmes pluripartites et sa recherche de la majorité électorale pour réaliser l'alternance au pouvoir. Parler donc de coalition ou d'alliance politique, c'est désigner une même réalité empirique qui consiste pour des acteurs de juger de la nécessité pour eux de se constituer temporairement ou permanemment en une force unique à travers une coopération partielle ou totale pour conquérir le pouvoir. Appréhendé ainsi, la coalition, à partir de son mode d'organisation et ses objectifs visent le changement social, et revêt un caractère revendicatif. Au regard de la fragilité et l'instabilité des regroupements politiques ivoiriens, en référence à la définition de Michelle et Karine, nous privilégierons dans notre article la notion de coalition à celle d'alliance.

\section{II.2. Approche théorique : la théorie du changement social}

Le changement social tel que défini par (Rocher, 2011) est un changement de structure qui s'inscrit dans le temps, en opérant une modification de l'organisation sociale dans sa totalité ou dans certaines de ses composantes significatives, telles que les conditions ou les modes de vie, ou

\footnotetext{
${ }^{9}$ Coalition électorale se situe au niveau des candidats, la coalition gouvernementale se situe au niveau des ministres et la coalition parlementaire concerne les députés.
} 
encore l'univers mental collectif. Ainsi définit, le changement s'inscrit dans une logique évolutionniste et peut dans certains domaines comme en politique, prendre un caractère révolutionnaire et idéologique. Défini ainsi, le changement social nous permet d'apprécier l'état de la démocratie en Côte d'Ivoire trois décennies après l'instauration du multipartisme.

\section{a. Le changement comme produit d'une révolution}

Pour parler de changement social, il est essentiel qu'on puisse indiquer les éléments structuraux ou culturels de l'organisation sociale qui ont connu des modifications et qui permettent de décrire ces modifications avec suffisamment de précision. En effet, on ne peut apprécier et mesurer le changement social que par rapport à une situation de référence dans le passé, à partir de laquelle on peut affirmer qu'il y a effectivement eu changement, indiquer ce qui a changé et dans quelle mesure ce changement s'est opéré. Le changement recherché peut présenter un caractère révolutionnaire soit par la substitution d'un système ou d'un régime politique nouveau à un ancien (Boudon ; Besnard ; Cherkaoui \& Lecuyer, 2012), soit par la rupture de la légalité résultante à la fois (1) de la restructuration profonde de l'espace politique en vue du renouvellement des acteurs politiques de premier rang; (2) de l'engagement politique de la société civile en faveur d'une éducation politique de la population.

Toutefois, quand la révolution est endogène, c'est-à-dire, suscitée et construite de l'intérieur sans influence d'acteurs extérieurs, elle sanctionne un mouvement de maturation du peuple. Par contre, lorsqu'elle vient de la volonté d'introduire de quelques manières que ce soit, un modèle étranger, ou bien, que les acteurs locaux recherchent toujours un appui extérieur pour la faire, comme c'est le cas dans de nombreux pays politiquement instables, on se trouve alors dans une forme de dirigisme politique de la part des acteurs derrière le voile. La rupture ainsi opérée par la révolution finit toujours par laisser réapparaitre et assez rapidement les continuités de l'ancien système.

\section{a- La forme évolutionniste du changement social}

Toutes les sociétés s'inscrivent dans une démarche commune qui évolue par phases basées sur l'opposition tradition et modernité. Le changement social obéit à cette logique et s'inscrit dans une dynamique évolutionniste qui implique nécessairement l'imperfection ${ }^{10} \mathrm{de}$ la reproduction des valeurs sociales, de la culture et des pratiques démocratiques caractéristiques des

${ }^{10}$ L'imperfection dans le processus de reproduction sociale est le fondement même de la théorie évolutionniste du changement social. 
individus de la génération précédente, transmises à ceux de la nouvelle génération (Harré, 1979). Le changement social, produit des interactions sociales, nécessite une double action agissante à savoir, la rupture avec les pratiques sociales non commodes et la continuité dans la reproduction sociale. Le président de la république Alassane Ouattara, par sa volonté de céder le pouvoir à une nouvelle génération en $2020^{11}$, s'inscrit de prime à bord dans cette démarche évolutionniste et décrit la nouvelle vision de la coalition.

\section{b. Changement social comme une idéologie}

Le changement n'étant pas exclusivement un phénomène extérieur à nous, en plus d'être une perception du monde, une certaine conviction, est aussi une idéologie ${ }^{12}$, mais, l'aspect idéologique du changement social est trop souvent négligé dans l'analyse sociologique (Rocher ; 1968). Allant dans le même sens, Grand'Maison (1970) relève l'implication d'idéologies nées de la volonté des hommes politiques dans les pays modernes de créer le changement par le renforcement de la démocratie. En effet, dans ces pays modernes, les hommes politiques, dans leur volonté de provoquer un changement et créer une société plus démocratique, expriment finalement chacun à travers ses terminologies, une idéologie du changement par la remise en question du modèle démocratique actuel, et faisant nécessairement appel à une transformation plus ou moins radicale.

Dans le contexte ivoirien dominé par la suprématie de «trois partis politiques ${ }^{13}{ } \gg$, qui sont les noyaux des coalitions, il n'est pas impertinent de s'interroger sur la capacité des partis politiques à opérer ce changement dans l'arène politique ivoirienne au regard de la survivance des reliques politiques dans leur fonctionnement telles que « l'absence ou la faiblesse du renouvellement et du rajeunissement des élites politiques, la prégnance du pouvoir gérontocratique qui empêche la visibilité de la jeunesse politique

\section{Analyse du cadre coalitionnel politique en Côte d'Ivoire III.1. La nécessité de former une coalition politique}

En Côte d'Ivoire, le choix opéré par les partis coalisés est d'aller tous aux au premier tour des élections et de soutenir le mieux placé parmi eux pour

\footnotetext{
${ }^{11}$ Discours du président ADO prononcé le 16 Juillet 2018 lors de l'assemblée générale constitutive du RHDP en un parti unifié dans lequel il a annoncé passer le pouvoir à la nouvelle génération, et former non pas un individu, mais une équipe pour sa succession dont le chef sera désigné de manière démocratique par l'ensemble des partis membres du RHDP.

${ }^{12}$ Rocher, G ; "l'idéologie est un système d'idées et de jugements, explicite et généralement organisé, qui sert à décrire, expliquer, interpréter ou justifier la situation d'un groupe ou d'une collectivité et qui, s'inspirant largement de valeurs, propose une orientation précise à l'action historique de ce groupe ou de cette collectivité »

${ }^{13}$ FPI ; PDCI ; RDR
} 
le second tour, du moins pour l'élection du président de la république, est une stratégie de maximisation de leur chance de remporter l'élection.

Cette stratégie indicatrice de la fin de l'ultra suprématie d'un parti dans l'arène politique ivoirienne, pose pour postulat que les coalitions politiques ont un pouvoir supérieur à la somme des pouvoirs de leurs membres (Le Bretony ; Karine; 2017).

La cessation de cette ultra majorité est une conséquence du système inclusif et concurrentiel instauré par le multipartisme avec la multiplicité des partis politiques qui a certainement fragilisé les "grands partis ${ }^{14}$. Cette fragilisation est marquée par la démission de certains de leurs militants pour adhérer à d'autres partis politiques. Elle a aussi favorisé une nouvelle configuration politique avec l'établissement d'un équilibre des forces électorales rendant quasi impossible pour un parti de remporter l'élection dès le premier tour. Par conséquent, la probabilité de gagner l'élection présidentielle ${ }^{15}$ est plus élevée au second tour à travers des coalitions.

Les résultats des élections présidentielles de 2000 et 2010 présentés dans les tableaux ci-dessous sont assez illustratifs de cet équilibre des forces électorales.

Tableau 4: Résultats des élections présidentielles de 2000 et 2010

\begin{tabular}{|c|c|}
\hline \multicolumn{2}{|c|}{ Election présidentielle de 2000 } \\
\hline Candidats & Suffrage exprimé en faveur des candidats au $1^{\text {er }}$ tour \\
\hline Laurent GBAGBO & $\mathbf{5 9 . 3 6 \%}$ \\
\hline Robert GUEI & $\mathbf{3 2 . 7 2 \%}$ \\
\hline Francis WODIE & $\mathbf{5 . 7 0 \%}$ \\
\hline MEL Eg Théodore & $\mathbf{1 . 4 7 \%}$ \\
\hline Nicolas DIOULO & $\mathbf{0 . 7 6 \%}$ \\
\hline
\end{tabular}

Taux d'Abstention : 62\% :

Taux de Participation : $35 \%$

\begin{tabular}{|c|c|c|}
\hline \multicolumn{3}{|c|}{ Election présidentielle de 2010 } \\
\hline Candidats & Suffrage exprimé au $1^{\text {er }}$ tour & $\begin{array}{c}\text { Suffrage exprimé } \\
\text { au } 2 \text { è tour }\end{array}$ \\
\hline Laurent GBAGBO & $38,04 \%$; FPI & $45,90 \%$, LMP \\
\hline Alassane OUATTARA & $32,07 \% ;$ RDR & $54,10 \%$, RHDP \\
\hline Henri Konan BEDIE & $25,24 \%$, PDCI & \\
\hline Mabri TOIKEUSSE & $2,57 \%$, UDPCI & \\
\hline Gnamien KONAN & $0,37 \%$, UPCI & \\
\hline Francis WODIE & $0.29 \%$, PIT & \\
\hline Siméon KONAN & $0.27 \%$, INDEPENDANT & \\
\hline Jacquelines LOHOUES Hobble & $0,26 \%$, INDEPENDANT & \\
\hline Pascal TOGOUA & $0,25 \%$, INDEPENDANT & \\
\hline Anaky KOBENAN & $0,23 \%$, MFA & \\
\hline Adama DOLLO & $0,13 \%$, INDEPENDANT & \\
\hline
\end{tabular}

${ }^{14}$ FPI-PDCI-RDR sont les trois partis majoritaires dans l'espace politique ivoirien

${ }^{15}$ Le code électoral ivoirien prévoit un second tour seulement pour l'élection à la magistrature suprême. 


\begin{tabular}{|c|c|c|}
\hline N'DOUBA Enoh Aka & $0,12 \%$, INDEPENDANT & \\
\hline Félix AKOTO Yao & $0,10 \%$, INDEPENDANT & \\
\hline Henry TOHOU & $0,05 \%, \begin{array}{l}\text { Union Socialiste des } \\
\text { Travailleurs }\end{array}$ & \\
\hline
\end{tabular}

Source : Françoise KAUDJHIS. O

La stratégie des coalitions d'éviter la candidature unique au premier tour s'explique par le fait que chaque parti membre veut se donner plus de chance d'être le représentant de la coalition au second tour, et traduit dans le même temps une faible coopération interne. Aussi, dans un contexte marqué par le manque de confiance aux institutions chargées des élections et la contestation des résultats pour motifs de fraude, elle vise à minimiser les fraudes au second tour. En effet, pour éviter d'être battue dès le premier tour, la coalition veut créer les conditions d'un second tour et se donner plus de chance de l'emporter. Ainsi, à partir des résultats du premier tour, les partis opposés à la conquête du pouvoir d'Etat jaugent leur chance d'obtenir la majorité des voix au second tour.

Les résultats des deux tours de l'élection présidentielle d'octobre 2010 montrent éloquemment l'équilibre des forces électorales et justifient la nécessité de la coalition comme stratégie de mobilisation d'électeur pour la conquête ou la conservation du pouvoir d'Etat. Les taux élevés de suffrage exprimé sans un écart considérable au premier tour de l'élection de 2010 sont attribués au FPI $(38,04 \%)$, RDR $(32,07 \%)$ et PDCI $(25,24 \%)$. En dépit des soutiens des alliés ${ }^{16}$ aux deux candidats (Laurent Gbagbo et Alassane Ouattara) qualifiés pour le second tour, il n'y a pas eu d'écart énorme prouvant l'ultra majorité du vainqueur $(\mathbf{5 4 , 1 0 \%}$ pour Alassane Ouattara et $\mathbf{4 5 , 9 0 \%}$ pour Laurent Gbagbo).

Les résultats de l'élection présidentielle d'octobre 2000 laissent apparaître un double constat : un taux élevé d'abstention (62\%) et un faible taux de participation (35\%) (Kaudjhis, 2001). Cela pourrait s'expliquer par la non-participation de deux des trois partis majoritaires de l'arène politique que sont le RDR et le PDCI pour raison d'invalidation des dossiers de candidature de leurs représentants. L'élection d'octobre 2000 à été la première la plus exclusive depuis 1990 , avec la rétention de 05 candidatures ${ }^{17}$ par la cour suprême $^{18}$ sur 19 dossiers de candidature. Au nombre des candidatures invalidées figuraient celles de Henri Konan Bédié, Emile Constant Bombé,

\footnotetext{
${ }^{16} \mathrm{Au}$ second tour, le candidat Alassane Ouattara du RDR a dans le cadre du RHDP bénéficié des voix de ses voix alliés: PDCI ; UDPCI ; UPCI ; MFA ; PIT. Le candidat Laurent Gbagbo du FPI a dans le cadre de LMP bénéficié des voix des partis politiques et candidats indépendants membres de LMP tels que RPP ; Adama DOLLO

${ }^{17}$ Guéi Robert ; Gbagbo Laurent ; Nicolas Dioulo ; Francis Wodié ; Mel Eg Théodore.

${ }^{18}$ Tia Koné président de la Cour suprême
} 
Lamine Fadiga (tous du PDCI ${ }^{19}$ et d'Alassane Ouattara du RDR. En effet, au regard de l'article 35 de la constitution de 2000, il était reproché à Henri Konan Bédié d'avoir présenté un dossier incomplet. L'examen médical prévu à l'article 35 de ladite constitution exige qu'un état complet de bien-être physique et mental soit dûment constaté par un Collège de trois Médecins désignés par le Conseil Constitutionnel sur une liste proposée par le Conseil de l'Ordre des Médecins de Côte d'Ivoire. Ces trois Médecins doivent prêter serment devant le Conseil Constitutionnel. Bédié ayant fait son examen médical en France, n'a donc pas respecté les exigences de l'article 35.

Quant à la candidature d'Alassane Dramane Ouattara, elle a été invalidée pour raison de «moralité douteuse » selon le président de la Cour suprême, qui reprochait un doute sur l'identité de ses parents. Ce doute qui affecte la validité de ses déclarations sur l'honneur de non renonciation à la nationalité ivoirienne et de non prévalence d'une autre nationalité ${ }^{20}$.

Trois décennies après l'instauration du multipartisme en Côte d'Ivoire, l'alternance démocratique pacifique recherchée par la stratégie coalitionnelle n'est pas effective et les coalitions nées ont toutes fait preuve d'une fragilité qui entame leur durabilité. Les échecs du Front Républicain, de la LMP et du RHDP assez illustratifs du caractère éphémère de ces coalitions, nécessitent l'identification et l'analyse des causes de ces échecs.

\section{III.2. Les causes de l'échec des coalitions politiques en Côte d'Ivoire}

Les coalitions politiques sont généralement filles des processus électoraux non inclusifs et non transparents avec leurs corolaires de crise de confiance et d'absence de consensus des acteurs politiques sur les institutions chargées d'organiser les élections.

Adoptée comme stratégie de mobilisation militante dans le but de se maintenir au pouvoir (parti au pouvoir) ou d'y accéder (parti d'opposition), ces coalitions sont confrontées à certaines pratiques qui concourent à leur échec. Il s'agit :

$>$ de l'absence d'inclusivité par le non respect du schéma inversé de la pyramide qui garanti la consultation préalable de la base.

$>$ du charisme trop fort des leaders politiques de la coalition ;

${ }^{19}$ Sur les 19 candidats, 9 étaient du PDCI.

${ }^{20}$ Kaudjhis : "Selon le président de ladite Cour, la génitrice de monsieur Ouattara nommée Nabintou Ouattara déjà décédée n'a pu devenir Nabintou Cissé encore en vie et que le mariage coutumier n'entraîne pas de changement de nom à l'état civil. Monsieur Ouattara était de nationalité voltaïque d'origine quand il était inscrit à l'université de Pennsylvanie et au moment de son mariage avec Dame Barbara Jean Davis à Philadelphie le 10 Janvier 1966, qu'il a été nommé Vice - Gouverneur de la BECEAO le ler Décembre 1982 et décoré la même année en qualité de Voltaïque » en conclusion de ne pas être "Ivoirien d'origine, né de père et de mère eux-mêmes ivoiriens d'origine " et de s'être "prévalu "à des moments de sa vie, de la Nationalité Burkinabé et donc d'être de moralité douteuse». 
$>$ de l'inexistence d'un projet de société commun clairement formalisé dès le départ entre les partis coalisés;

$>\mathrm{du}$ comportement nombriliste des partis politiques membres de la coalition qui nourrissent des projets personnels inavoués.

\section{a. L'échec du Front Républicain.}

$>$ L'absence d'un projet de société commun clairement formalisé dès le départ entre les partis coalisés

Un parti politique en tant que groupement considéré d'utilité publique, se crée sur la base d'une vision, d'une idéologie, qu'il matérialise dans un projet de société qui lui sert d'argument de campagne électorale pour la conquête du pouvoir. Tout parti politique se crée donc sur la base d'un projet de société qui lui propre. Partant de ce fait, il est donc quasiment impossible pour des partis politiques opposés idéologiquement de décider de créer une coalition durable tout en restant chacun dans son courant politique originel.

A la suite de l'élection d'Henri Konan Bédié en 1995, la situation sociopolitique s'est davantage précarisée avec le caractère " ethniciste » que prend l'opposition entre Alassane Dramane Ouattara et Henry Konan Bédié. Cette ethnicisassion a dégradé les rapports sociaux entre le groupe Akan dominé par l'ethnie baoulé et le groupe ethnique Mandé nord dominé par l'ethnie malinké communément appelé « Dioula» (Bouquet, 2005). Face à l'ambition politique du leader du RDR qui de plus en plus représentait une menace politique pour le PDCI, ce parti entreprend une vaste campagne pour prouver à la communauté nationale et internationale qu'il est étranger, et justifier son exclusion de la compétition électorale à la magistrature suprême. A cet effet, une disposition a été introduite dans le code électoral qui a soutenu le concept «d'ivoirité » pour distinguer le vrai ivoirien du faux (Bouquet, op cit.). Le concept d'ivoirité, au lieu d'être une conception positive de la nationalité ivoirienne favorable à l'unité nationale, sous l'effet des diverses interprétations à des fins politiques, a été la cause de l'accentuation du clivage entre les populations. La campagne pour la discréditation du candidat du RDR à l'effet de l'éliminer de la compétition électorale (Gobille, 2009) a contribué à sa popularité et l'a désormais inscrit sur la liste des leaders politiques charismatiques en le propulsant au-devant de la scène politique comme le symbole de l'exclusion sociale et politique, à laquelle se sont progressivement identifiées les populations du nord, qui avaient le sentiment d'être devenues des citoyens de seconde zone (Akindes ; Fofana \& Koné, 2010).

Ce contexte a favorisé le rapprochement des deux principaux partis de l'opposition d'alors qu'étaient le RDR d'idéologie libérale et le FPI d'idéologie socialiste pour former en 1995 une coalition nommée «Front Républicain ». L'unique objectif de cette coalition était d'évincer Henry Konan Bédié du pouvoir. 
Toutefois, fonctionnant au gré de l'évolution de la situation politique et des opportunités qu'elle offrait, cette coalition présentait déjà des signes d'effritement de par sa fondation et son objectif. Unis uniquement pour évincer le PDCI du pouvoir, les deux partis n'ont pu avoir une vision à long terme pour la consolidation et la durabilité de leur coalition. En effet, lorsque des partis opposés idéologiquement, et tous engagés à la conquête du pouvoir, décident pour la circonstance de s'unir seulement pour conquérir le pouvoir, la probabilité que leur union se dissolve est plus élevée une fois l'objectif atteint. Au sein de telles coalitions on assiste plus à la prégnance des rapports de force au détriment des rapports juridiques entre les partis coalisés (Duverger, 1954).

Après le coup d'Etat du 24 décembre 1999, la coalition s'est immédiatement estompée à cause de l'opportunité de conquête du pouvoir qu'offrait ce coup d'Etat à chacun des partis. Ce comportement opportuniste est la conséquence de l'absence d'un projet de société formalisé par les partis coalisés dès le départ. Un tel projet ne pouvait être possible qu'à condition qu'il y ait une fusion totale des partis, ou que l'un d'eux acceptait de renoncer à ses objectifs et soutenir l'autre. Mais, l'opportunisme du moment ne pouvait permettre l'existence de ce projet. A l'approche de l'élection présidentielle de 2000 le climat sociopolitique s'est fragilisé davantage avec l'exclusion annoncée du candidat du RDR à la suite des résultats du référendum de juillet 2000 qui ont donné « 86,53\% de « OUI » contre 13,47\% de «NON » pour la nouvelle constitution ${ }^{21}$. Le climat pré-électoral était donc plombé par la question des conditions d'éligibilité, notamment celle du candidat du RDR. C'est ainsi que l'article 35 de la nouvelle constitution soumise à référendum sur lequel les ivoiriens devaient se prononcer dans les urnes en faisant le choix entre les conjonctions de coordination ET/OU qui doit y être introduite, va polariser l'attention, (Bastart, 2014-Bouquet, 2005). Selon cet article, le président de la république doit être ivoirien d'origine, né de père et de mère eux-mêmes ivoiriens d'origine. Sur la base de cette clause qui doit être introduite, la population ivoirienne était appelée à faire le choix entre : (i) le futur président de la République doit être né de père $\mathbf{E T}$ de mère ivoiriens de naissance; et (ii) le futur président de la République doit être né de père $\mathbf{O U}$ de mère ivoiriens de naissance. Après de multiples revirements, le "ET" fut finalement inscrit dans le texte. Avec le soutien que le FPI et son président Laurent Gbagbo apportaient aux résultats de référendum, la rupture entre les partis coalisés du front républicain était désormais consommée.

${ }^{21}$ Référendum constitutionnel ivoirien de 2000-Wikipedia 


\section{b. L'échec de La Majorité Présidentielle (LMP). Le leadership dominant de Laurent Gbagbo et l'inexistence d'un projet commun clairement formalisé dès le départ entre les partis coalisés.}

Après l'élection de 2010 sanctionnée par la victoire d'Alassane Ouattara et l'arrestation du Président Gbagbo le 11 avril 2011 suivi de son transfèrement à Cour Pénale Internationale la même année, on a assisté à l'extinction de la LMP. L'une des causes explicatives de son extinction est le leadership dominant de son leader. En effet, le président Laurent Gbagbo a eu une double représentation symbolique au sein de cette coalition. Il y a représenté à la fois le chef et le leader. Cette double casquette n'a pas été favorable à sa pérennisation.

Avant d'aborder l'analyse de ce constat, nous allons lever un voile sur la confusion qui est faite par de nombreuses personnes entre le chef et le leader. Dans un groupe social, il peut y avoir un chef et un leader, mais aussi il peut avoir un individu qui soit à la fois le chef et le leader. Olivier Meier (2019) s'appuyant sur l'analyse de Max Weber fait une nette distinction entre ces deux concepts. Dans son approche de la notion de chef, il la renvoie à la légalité et au principe de la hiérarchie par l'établissement de rapport horizontal entre le chef et ses subordonnés. L'autorité exercée par le chef est légale, car ceux sur elle s'exerce, obéissent en réalité aux lois qui lui confèrent son autorité. Dans ce genre de cas, le fondement de la légitimité de l'autorité du chef se trouve dans le respect des lois et repose sur la croyance en la légalité des règlements arrêtés et du droit de donner des directives. On se trouve dans une organisation au sein de laquelle les membres obéissent à un ordre impersonnel légalement arrêté, et aux supérieurs désignés par cet ordre, en vertu de la légalité formelle de ses règlements.

La notion de leader quant à elle renvoie moins au principe de la légalité qu'à celui de la légitimité. L'autorité exercée par le leader tire vient de son charisme $^{22}$ et de son aura. Les valeurs qu'il incarne qui sont profitables et appréciées par les membres du groupe sont donc le fondement du pouvoir du leader.

Pour plusieurs analystes, l'autorité individuelle du leader politique serait l'élément essentiel de la cohésion du parti et de l'adhésion des membres, ce qui favorise la personnalisation de certains partis politiques (Duverger, 1968 ; Hervé, 2016) $)^{23}$ qui dans leurs fonctionnement ne reposent que sur le

${ }^{22}$ Le charisme au sens de Max Weber désigne une qualité considérée comme extraquotidienne au nom de laquelle une personnalité est regardée comme un chef. Le charisme est donc fondé sur une relation sociale entre un porteur de charisme et ses collaborateurs qui croient en son charisme.

${ }^{23}$ Hervé en référence à la typologie des partis politiques africains établie par Sébastien Elischer: les partis mono-ethniques, les alliances politiques multiethniques, les partis attrape- 
charisme de leurs leaders, comme ce fut le cas de la LMP qui reposait en réalité sur la personnalité de son leader Laurent Gbagbo.

L'extinction de la LMP comme nous l'avons déjà dit, s'explique par l'influence du leadership de Laurent Gbagbo. En effet, depuis son arrestation et son transfèrement à la CPI, on a constaté une division au sein de son parti d'origine (FPI) chef de fil de cette coalition mettant en face la tendance Affi Pascal N'guessan et les pros tendance Laurent Gbagbo appelés « les GOR) ${ }^{24}$ » qui n'ont de référant politique que lui bien qu'il soit hors du pays.

Son parti d'origine n'arrive pas à trouver un leader à sa dimension, capable de garantir son unité. Bien qu'Affi N' guessan Pascal ait été reconnu par la justice ivoirienne comme le président légal du FPI, il n'arrive pas à créer cette unité. Ses propos relatifs à la dissidence au sein du parti mettent clairement en évidence son incapacité à unifier le parti, et indiquent Laurent Gbagbo comme la figure symbolique de son unité.

"D'abord il faut noter que le retour du président Gbagbo va mettre fin à la crise, ça c'est la première chose importante pour le FPI parce que tout compte fait, la crise se cristallise autour de la personne du président Laurent Gbagbo. La libération de Laurent Gbagbo et de Blé Goudé va renforcer l'unité au sein du parti et d'appréhender 2020 avec davantage d'assurance et de sérénité ». (Pascal Affi N'guessan)

L'extinction de la LMP est indicatrice de la faiblesse du lien entre les partis membres. En effet, dans le contexte de sa formation, l'enjeu était de démontrer sa popularité et sa majorité face au RHDP. A cet effet, toutes les demandes d'adhésion étaient acceptées. Les mobilisations au cours des meetings politiques se faisaient sur la base des promesses comme l'indique les propos ci-dessous du responsable de l'unesci. Les médias d'Etat étaient plus utilisés pour mettre en visibilité ces adhésions et mobilisations pour faire la preuve de sa majorité.

"Les gars nous sommes invités au meeting du président Gbagbo à Dabou, mobilisons nous tous pour y être. Je sais que nous sommes de bords politiques différents, chacun de nous à son parti, mais je demande à chacun de prendre les dispositions pour y être dans l'intérêt de notre organisation. Nous devons tous être ici au siège ce jour à $8 H$ pour le départ. Actuellement nous avons des difficultés de fonctionner par manque de moyens financiers. Nous avons fait une Réunion chez le président Mel, en tout cas, il a mis à notre disposition les moyens pour faire une bonne mobilisation. Je lui ai promis au

tout multiethniques, les partis programmatiques et les partis reposant sur la personnalité de leur leader ${ }^{24}$ GOR=Gbagbo Ou Rien 
moins deux gros cars pour ce meeting ». (Responsable d'un syndicat d'élève et étudiants : UNESCI) ${ }^{25}$

A ces meetings se trouvaient à la fois des partisans, des sympathisants, des personnes curieuses, des personnes venues à cause des tee-shirts, des personnes en âge de voter et celles qui n'ont pas l'âge de voter. Face à l'enjeu de la majorité, cette stratégie moins réaliste a été fortement exploitée par LMP. Convaincus de sa victoire qu'elle exprime à travers son slogan de campagne « on gagne ou on gagne », elle n'a pas eu de vision à long terme de sa pérennisation en cas de perte. Sur le plan organisationnel, elle présentait des faiblesses qui présageaient son extinction. Structurée autour du FPI qui en était le noyau, elle comptait une soixantaine d'organisations composées de certains partis politiques non signataires des accords de Linas-Marcoussis, de certaines organisations de la société civile, des groupes de soutien et de mobilisation. Les adhésions à la LMP ne se faisaient pas sur la base d'un projet de société commun produit par les membres et qui les engage tous. Elles se faisaient librement sans engagement formel, à condition que l'adhérant manifeste son soutien au référant politique Laurent Gbagbo. Une coalition créée sur cette base présente des failles dans son dispositif organisationnel et est plus exposé à la disparition après son leader.

\section{c. La fragilité du Rassemblement des Houphouétistes pour la Démocratie et la Paix (RHDP).}

La fragilité du Rassemblement des Houphouetiste pour la Démocratie et la Paix (RHDP) est la conséquence (1) du non-respect par les partis membres du principe d'inclusivité qui obéit au schéma inversé de la pyramide dans la prise de décision pour la mise en place des coalitions; (2) de l'inexistence d'un projet commun clairement formalisé dès le départ entre les partis coalisés; (3) du comportement nombriliste des partis politiques membres de la coalition qui nourrissent des projets personnels inavoués.

Composé de six partis politiques ${ }^{26}$ à sa création le 18 Mars 2005, avec pour objectif de constituer une majorité électorale face à La Majorité Présidentielle, le RHDP a été transformé en un parti unifié le 16 Juillet 2018. $\mathrm{Au}$ regard de l'histoire politique des leaders des deux partis majoritaires (PDCI et RDR) de cette coalition, marquée par des violences extrêmes entre leurs militants, plusieurs acteurs de l'opposition politique ne croyaient pas en

\footnotetext{
${ }^{25}$ Diarassouba, Thèse de doctorat, 2016

${ }^{26}$ Mouvement des Forces d'Avenir (MFA) ; Parti Démocratique de Côte d'Ivoire (PDCI) ; Parti Ivoirien des Travailleurs (PIT) ; Rassemblement Des Républicains (RDR) ; Union pour la Démocratie et la Paix en Côte d'Ivoire (UDPCI) ; Union pour la Paix en Côte d'Ivoire (UPCI)
} 
sa longévité. Le président Laurent Gbagbo s'y moquait en ces termes « la fille violée tombée amoureuse de son violeur ».

Bien que cette coalition ait vécu plus longtemps que les autres ${ }^{27}$ (15ans), mais, avant la fin du premier mandat du président Alassane Ouattara, sont apparus les signes annonciateurs de sa fragilité. Le premier signe était la négociation pour un second mandat qui a abouti au fameux «appel de Daoukro » que j'appelle «l'appel du malaise au sein du RHDP». Pour rappel, les partis membres du ce regroupement avaient décidé de renforcer leur union dans le but de conserver durablement le pouvoir d'Etat. Dans cette perspective, le 17 septembre 2014, Henri Konan Bédié a sollicité l'union de tous les partis membres autour de la candidature unique d'Alassane Ouattara à l'élection présidentielle de 2015 à travers cet « appel de Daoukro».

"J'ai accepté en 2015 au prix de nombreux sacrifices de ne pas présenter de candidature au titre de mon parti. Les termes de cet appel sont clairs. L'objectif, je le répète était de maintenir le pouvoir dans nos rang, convaincu que le travail colossal effectué par le président Alassane Ouattara, avec un second mandat à la tête du pays remportera d'autre victoire pour le bonheur des ivoiriens ». (Discours de Bédié à Daoukro le 17 septembre 2017).

La négociation pour un second mandat prend tout son sens au regard de la promesse du président Alassane Ouattara pendant sa campagne électorale de ne lui accorder qu'un seul mandat (5ans) pour la mise en œuvre intégrale de son programme de gouvernement avec in fine, la création d'un million d'emplois. Ayant constaté l'impossibilité de réaliser son programme électoral durant ce premier quinquennat ${ }^{28}$, il négocie un second mandat en vue de continuer son œuvre de développement entamée mais inachevée. Pour se donner plus de chance d'être réélu en 2015 il négocie avec le président du PDCI, Henri Konan Bédié pour être l'unique candidat du RHDP à l'élection présidentielle d'octobre 2015 d'où l'appel de Daoukro lancé par Henri Konan Bédié le 17 septembre 2014 aux partis membres et à leurs militants d'accepter cette candidature dans le but de maintenir le pouvoir.

Etant donné qu'il n'ya pas d'acte désintéressé en politique, il est donc pertinent se s'interroger sur ce que gagne le PDCI, conscient de son poids politique au sein du RHDP, à renoncer à la présentation d'un candidat en 2015 conformément à la décision issue de son congrès politique de 2013. Bien

\footnotetext{
${ }^{27}$ Quinze ans en 2020 au moment de la rédaction de l'article

${ }^{28}$ Le président justifie l'impossibilité d'atteindre son objectif durant son premier quinquennat parce qu'il a hérité d'un Etat où tout était à reconstruire à cause des affres de guerre postélectorale. L'urgence pour lui était la reconstruction du pays avant l'application de son programme tel que conçu avant les élections.
} 
évidemment, cette renonciation a certainement créée un nouveau cadre de renégociation des accords précédents en termes de coût-avantage pour lui. Il est bon de rappeler à toute fin utile que cette décision non conforme aux décisions du congrès politique n'était pas partagée par le président de la jeunesse Kouadio Konan Bertin (KKB). Dans une logique conformiste, il demandait l'application de ces décisions en présentant un candidat en 2015 pour la reconquête du pouvoir. Face au refus du bureau politique, il se retire et se présente en tant que candidat indépendant à l'élection présidentielle de 2015.

"(......).Au dernier congrès politique du parti, vous devez le savoir, le congrès c'est l'instance suprême d'un parti comme le PDCI, et c'est au congrès politique du PDCI qu'on prend toutes décisions importantes qui engagent la vie du parti. En 2013, il y a donc eu un congrès au parc des sports de Treichville et à l'unanimité des militants réunis ce jour-là nous avons décidé de nous donner un candidat en 2015. On a même ajouté un terme de militant actif pour nous représenter aux élections présidentielles. A partir de là, je m'attendais comme tout le monde à ce que le parti se mette en branle pour reconquérir le pouvoir quand l'appel de Daoukro est venu prendre le contre-pied de ce qu'on a décidé ». (Kouadio Konan Bertin, membre du bureau politique du PDCI)

Sachant que tous les accords se tissent sur la base des calcul-coût et avantage des membres de la coalition, négocier un second mandat en s'appuyant sur le PDCI, est déjà la remise en cause tacite des accords précédemment conclus, et certainement la renégociation de nouveaux accords ou des accords complémentaires. Cet appel au départ salutaire parce que considéré comme salvateur, a fini par être la source de discorde entre les partis houphouetistes, car il aurait prévu et garanti l'alternance du pouvoir entre les partis houphouetistes avec la cession du pouvoir d'Etat au PDCI en 2020.

«Notre alliance résout le problème de la gestion du pays et l'appel de Daoukro balise le problème des successions au pouvoir. Chacun sait qu'il est désormais impossible à une formation politique si puissante soit elle de gérer notre pays. C'est la raison pour laquelle au niveau de notre alliance nous devons nous accepter les uns les autres et trouver entre nous les moyens de gérer ensemble la Côte d'Ivoire ». (Discours de Bédié à Daoukro le 17 septembre 2017).

Toutefois, la non formalisation de cette alternance au profit du PDCI en 2020 a été la cause de la discorde au sein du parti des houphouetistes. Ainsi, sont opposés, comme l'illustrent les propos ci-dessous, ceux qui s'inscrivent 
dans une démarche de désignation consensuelle du candidat ${ }^{29}$ unique du RHDP en 2020 sur la base d'un critérium, et ceux qui réclament un devoir de reconnaissance ${ }^{30}$ pour son soutien au Président Alassane Ouattara en 2010 et 2015. Cette reconnaissance qui devrait se traduire par un consensus des houphouetistes autour de la candidature de son représentant en 2020 pour le compte du RHDP.

"L'alternance en 2020 n'est pas négociable. Aujourd'hui le président Bédié a soutenu son jeune frère en 2010. Son jeune frère a fait un travail excellent et il se trouve que ce travail n'est pas terminé, et le président Bédié dit, nous nous sommes mis ensemble pour faire un mandat RHDP, mais si nous devons avoir un candidat PDCI opposé à un candidat RDR, quel va être l'élément essentiel de cette campagne? Mettons-nous ensemble et accompagnons le champion qui gagne. Mais en même temps nous pensons qu'en 2020 qu'il y'ait l'alternance et que le pouvoir d'Etat revienne à un cadre du PDCI. ». (Coulibaly Zié Daouda, membre du Bureau politique du PDCI-RDA, entretien sur son livre «le sens de l'appel de Daoukro »

"[....] Le RDR n'est pas venu au pouvoir seulement pour dix ans; un pouvoir qui a été exercé par d'autres partis en 40 ans, en 50 ans dans ce pays. Quand je disais cela, on disait que j'avais un discours politiquement incorrect. Moi j'assume tous mes discours, j'assume toutes mes interviews, j'assume tous mes actes, tous. Parce que moi je suis engagé, je sais ce que veut dire l'engagement, la conviction. L'engagement et la conviction ne s'embarrassent pas de fioriture. La conviction et l'engagement ne s'embarrassent pas de peur [....].Des gens se lèvent matin, midi et soir pour parler de l'alternance en 2020. On dit alternance, c'est-à-dire "toi, il faut te lever je vais m'asseoir ». Est-ce que c'est ça on appelle un offre politique? Ça, ce n'est pas une offre politique. (...) Alors que qu'on est assis ensemble, on mange ensemble. Toi-même tu manges plus que moi, tu es devenu clair, tu es devenu rond, tu brilles, parce que tu n'as aucune charge. On te donne seulement tu manges. Tu n'arrives même pas respirer correctement. Maintenant, toi tu dis "lève-toi" alors qu'on mange ensemble. Pourquoi je vais me lever? Ça veut dire que tu veux manger seul ». (Cissé Ibrahima Bacongo, meeting à Adjamé le 16 aout 2017).

${ }^{29}$ Les militants du RDR

${ }^{30}$ Les militants du PDCI 
L'espoir du PDCI de voir sa candidature soutenue par l'ensemble des partis membres du RHDP s'amenuise considérablement avec le souhait du président Alassane Ouattara de faire prévaloir la démocratie à travers le choix consensuel du candidat de la coalition.

«(...). J'ajoute que l'élection présidentielle de 2015 a été totalement démocratique. A cet égard, je remercie mes adversaires qui m'ont téléphoné pour me féliciter. J'ai été très fier de la Côte d'Ivoire, de mes compatriotes et surtout des Partis politiques qui ont eu cette élégance. Je souhaite qu'en 2020, ce soit la même chose. Et surtout qu'au sein du RHDP, nous fassions jouer la démocratie et que tous les enfants d'Houphouët-Boigny, dans tous les Partis politiques, se réunissent pour désigner un seul candidat comme Président de la République et un seul candidat comme Vice-président de la République ». (ADO, discours à l'assemblée constitutive du RHDP parti unifié, juin 2018)

Le PDCI accuse le RDR de ne pas respecter l'accord « tacite » conclu entre eux lors de l'appel de Daoukro. Selon les militants du PDCI l'appel de Daoukro a été conditionné par la garantie de la cession du pouvoir d'Etat à leur parti en 2020. Nous qualifions donc cet accord de tacite parce qu'à la lecture de l'appel de Daoukro, la cession du pouvoir au PDCI en 2020 n'y est mentionnée nulle part. L'inexistence formelle de cet accord fait qu'il est diversement interprété ou non reconnu au sein du RHDP concernant la succession d'Alassane Ouattara en 2020.

Face à l'ambigüité de la position du président Alassane Ouattara sur sa candidature en 2020, et les discours des militants du RDR l'encourageant à briguer un autre mandat ${ }^{31}$, le PDCI adopte alors la stratégie de la prudence par le report de son congrès d'adoption ou pas des statuts du RHDP unifié après l'élection présidentielle de 2020. Cette prudence lui permettrait de présenter son candidat au cas où celui qui sera choisi pour le compte du RHDP ne sortirait de ses rangs.

\section{d. Le non-respect par les partis du principe d'inclusivité dans la prise de décision pour la mise en place des coalitions.}

Le principe d'inclusivité à travers la consultation de la base et la prise en compte de son avis est indispensable au projet de coalition et nécessaire à sa consolidation et sa durabilité. Le non respect de ce principe par les coalitions favorise l'affaiblissement des liens entre ses membres par le développement de la coopération / la concertation au détriment de la

\footnotetext{
${ }^{31}$ Un débat est ouvert à ce sujet au regard de la constitution du 08 Novembre 2016 qui consacre la troisième République.
} 
coordination (favorable à un changement). Bien qu'étant d'accord sur la nécessité de s'unir pour conquérir le pouvoir ou pour s'y maintenir, toutefois, dans leurs engagements ils sont tiraillés entre la logique des intérêts partisans et la logique d'intérêt de la coalition. Ce principe n'a pas été adopté par les partis fondateurs du RHDP. Sa mise en place a été décidée par les présidents des partis membres sans préalablement prendre en compte les aspirations de leur base. En effet, c'est en Mai 2005 à Paris que les leaders du PDCI, RDR, MFA et UDPCI ont décidé d'unir leur force contre le parti au pouvoir dans le but d'assurer la victoire aux prochaines élections. Ils ont été rejoints plutard par d'autres partis notamment l'UPCI, le PIT.,

«Nous étions en 2003 dans une situation particulière en Côte d'Ivoire surtout sur la scène politique. Il y avait beaucoup de doute. (...). Nous nous sommes dit pourquoi les enfants d'Houphouët Boigny, les animateurs de tous les partis Houphouëtistes ne retrouvaient pas pour se donner les moyens de faire renaître cette Côte d'Ivoire là. Il y avait des sacrifices à faire il y avait surtout à bâtir cette confiance là de sorte à respecter les engagements qui étaient contenus dans la plateforme de Paris notamment se soutenir mutuellement, préparer les élections ensemble, de faire en sorte que si à l'issue du premier l'un de nous arrivait au second tour nous nous mobilisons à soutenir sa candidature, et c'est ce que nous avons fait, et après il fallait défendre cette victoire, après il fallait gouverner ensemble ». (Albert Mabri

Toikeusse, Président de l'UDPCI)

\section{e. L'inexistence d'un projet commun clairement formalisé dès le départ entre les partis coalisés}

Les dissensions au sein des coalitions naissent dès que l'objectif d'accession au pouvoir exécutif est atteint, avec l'entrée en jeux des opportunités de positionnement politique qui se présentent aux partis membres. Ces dissensions sont la conséquence de l'absence d'un projet de coalition formel qui engage les membres et régule leur rapport. Certes, l'existence de ce projet n'est pas une garantie suffisante de réussite de la coalition, mais elle crée une confiance qui, tant qu'elle est maintenue et garantie l'intérêt des membres, favorise la cohésion et la bonne coopération entre ses membres, indispensables pour la durabilité de la coalition. La durabilité et solidité d'une coalition politique reposent sur un projet coalitionnel savamment élaboré et formalisé, qui favorise l'engagement effectif de chaque membre. Toutefois, tant que les accords entre les partis membres resteront verbaux, ils peuvent faire l'objet de sa non reconnaissance par certains membres comme ce fut le cas au RHDP, ce qui expose la coalition à un risque élevé de désintégration. 
En dépit de la solidité apparente que ne cessent de mettre en avant les membres du RHDP, les remous suscités après le choix de son candidat unique en la personne de l'ancien premier ministre Amadou Gon Coulibaly, illustrent bien sa fragilité. En effet, après la décision d'Alassane Ouattara de ne pas se présenter aux élections de 2020 et laisser la place à la nouvelle génération du nouveau parti unifie ${ }^{32}$, chaque membre qui s'inscrivait dans cette catégorie de nouvelle génération voyait en cette décision une chance d'être désigné candidat et chef d'équipe de la coalition. Mais, en nous référant au discours du président de l'UDPCI à la réunion du conseil politique du RHDP du 12 Mars 2020 pour le choix de son candidat, le choix d'Amadou Gon Coulibaly n'a semblé pas faire l'objet d'un consensus.

«(....). Monsieur le président, j'ai donc à vous dire que vous êtes le fils d'Houphouet Boigny et Houphouët nous a enseigné que tout ce qui peut opposer les hommes fini toujours par le dialogue, et il recommandait que nous fassions usage du dialogue pour éviter donc que ces situations surviennent ou s'aggravent. (....). Donc monsieur le président, faites comme votre père. Continuez donc dans le dialogue à rassembler au sein du RHDP, à rassembler autour du grand projet de nous mettre en équipe après vous et que nous ayons l'occasion de poursuivre victorieusement le travail que vous avez bien commencé». (Albert Mabri Toikeusse, lors de la réunion du conseil politique du RHDP du 12 Mars 2020 pour le choix de son candidat à l'élection d'octobre $2020^{33}$ )

Plusieurs partis souffrent de l'influence de leurs leaders dans le choix des militants à des postes de responsabilité. Ils pèsent de tout leur poids lors des congrès électifs pour le choix des autres dirigeants et par peur de perdre des avantages, les autres membres n'osent pas contester leurs choix. Pour ceux qui s'y aventurent en prenant le risque de perdre leurs avantages finissent en cas d'échec par démissionner et rejoignent d'autres partis de l'opposition souvent en collaboration avec d'autres compagnons déserteurs (Issiaka, 2017).

A la suite du décès d'Amadou Gon Coulibaly le 08 juillet 2020, suivi ce même mois de la démission du vice-président Daniel Kablan Duncan, certains membres n'ont eu autre solution que de demander au président Alassane Ouattara de reconsidérer sa décision et accepter d'être candidat. La difficulté de trouver un nouveau candidat qui fasse l'objet d'un consensus illustre bien le malaise sur fond de crise de confiance en son sein tel qu'exprimé par le président.

\footnotetext{
${ }^{32}$ Le RHDP est passé d'un regroupement de partis en un parti unique

${ }^{33}$ Allocution à la réunion du conseil politique du RHDP du 12 Mars 2020 pour le choix de son candidat à l'élection présidentielle d'octobre 2020.
} 
"Si je n'avais pas accepté, je crains fort que le RHDP aurait connu une désintégration. J'ai accepté de me présenter par obligation. L'année prochaine j'aurais 80 ans et tous ceux de mon âge ici savent que travailler $12 \mathrm{~h}$ à $14 \mathrm{~h}$ par jour à 80 ans ce n'est pas facile. Mais je l'ai fait pour la paix ». (ADO devant les cadres et guides religieux dans le cadre de sa visite dans le district de la savane au journal télévisé de $20 \mathrm{H}$ du 10 octobre 2020).

Ces propos du chef de l'Etat font appel à un double constat. Primo, le risque de désintégration auquel était exposé le parti s'il n'avait pas accepté de reconsidérer sa décision est certainement un aveu d'échec tacite de sa part pour la formation d'une équipe qui va assurer la continuité. Secundo, ils confirment que le choix du premier candidat n'a pas été fait sur une base consensuelle, et que ce choix ne bénéficiait pas certainement de l'assentiment de certains cadres de la coalition. Faire appel à la reconsidération de sa décision en acceptant d'être candidat comme l'unique solution, démontre que le RHDP est en réalité très fragile et court le risque de se disloquer ou même de disparaitre après le président Alassane Ouattara.

"Après la survenue du drame (décès du premier ministre amadou Gon Coulibaly), la majorité des militants s'est tournée vers le président Alassane Ouattara. Il est la solution aujourd'hui, il est notre solution et je le lui ais exprimé (....). En l'état actuel oui, il est le bon candidat le mieux placé pour battre le candidat Bédié au premier tour. Il est aujourd'hui le ciment de notre union, et au sein du RHDP, il est notre refuge. Il est en même temps le catalyseur de toutes nos ambitions (...), voilà, c'est pour éviter la guerre des dauphins en notre sein, et mieux se concentrer sur l'élection du 31 octobre 2020 (...) ». (Adama Bitogo, Directeur exécutif du RHDP, entretien sur la RFI le 20 juillet 2020)

Sa décision de porter la candidature de son parti a certes été salvatrice pour ses partisans, mais n'a pas manqué de susciter les manifestations de l'opposition qui, au regard de la constitution qualifie cette autre candidature d'illégale. Ainsi, s'engage un débat juridique sur la légalité ou pas de cette candidature, sur lequel il n'y a pas de positions tranchées des juristes constitutionalistes. Pour certains (favorables à cette candidature), vu que la nouvelle constitution consacre la troisième République, alors, au nom du principe de la non rétroactivité de la loi, il ne peut s'agir d'un troisième mandat, mais, d'un premier mandant de la troisième République.

Pour d'autres (opposés à cette candidature), bien que la constitution consacre la troisième république, il s'agit bien d'un troisième mandat, car, à la lecture de l'article 183 de la constitution relatif à la continuité législative qui stipule que : «La législation actuellement en vigueur en Côte d'Ivoire reste 
applicable, sauf l'intervention de textes nouveaux, en ce qu'elle n'a rien n'a de contraire à la présente Constitution », la limitation du mandat à 2 est maintenue, par conséquent il s'agit bien d'un troisième mandat.

Sans nous lancer dans un débat juridique sur cette question, ce qui fera certainement l'objet d'une prochaine publication, il est bien de noter que cette situation a, d'une part, occasionné le retrait de certains cadres du RHDP pour rejoindre l'opposition, d'autre part, favorisé le rapprochement de plusieurs partis d'opposition pour constituer face au RHDP une force électorale nommée Coalition pour la Démocratie, la Réconciliation et la Paix (CDRP).

\section{f. Le comportement nombriliste des partis politiques membres de la coalition qui nourrissent des projets personnels inavoués}

Généralement, les discussions entre les différents acteurs de l'opposition pour la formation d'une coalition électorale au premier ou au second tour d'une élection présidentielle, s'articulent autour des bénéfices sur lesquels ils pourraient miser comme «butin de victoire » de leur union. Ces bénéfices sont généralement les postes au gouvernement et dans l'administration, y compris les structures parapubliques. Mais, il existe souvent une crise de confiance entre les membres de la coalition comme c'est le cas au RHDP quant à la garantie que le « candidat unique » va honorer les promesses électorales une fois au pouvoir. Au RHDP, on a assisté à des logiques différentes mettant en face les militants du RDR qui souhaitent conserver le pouvoir en passant outre les accords, et d'autres partis membres pour qui veulent conquérir le pouvoir d'Etat en 2020. Ces comportements opportunistes ont mis en avant l'intérêt particulier des partis au détriment de l'intérêt général de la coalition.

\section{Conclusion}

Depuis l'adoption du multipartisme en 1990 par la Côte d'Ivoire, la coalition à travers des alliances politiques est la nouvelle stratégie des partis politiques pour la conquête du pouvoir d'Etat. Débuté par le front républicain en 1995 entre le RDR et le FPI, s'en sont suivis le RHDP en 2005 et LMP en 2010. De ces trois coalitions, seul le RHDP existe. Toutefois, en dépit de sa relative longévité comparativement aux autres coalitions, il fait face au défi de sa stabilité, avec la prégnance de la question de l'alternance en interne. Les remous suscités après le choix par le Président Alassane Ouattara de l'ancien premier ministre Amadou Gon Coulibaly comme le candidat du RHDP, illustrent bien l'instabilité au sein de cette coalition. Aussi, la difficulté de trouver un nouveau candidat qui fasse l'objet d'un consensus après le décès d'Amadou Gon Coulibaly le 08 juillet 2020, au point que le président Alassane Ouattara reconsidère sa décision (de ne pas se présenter) et se porter candidat, traduit bien le malaise sur fond de crise de confiance au sein de cette coalition. 
Au regard de ce constat, il se pose la question de leur capacité à réaliser le renouveau démocratique en Côte d'Ivoire à travers un changement politique pacifique.

Dans le cadre de cet article qui est une réflexion sur les causes des échecs de ces coalitions politiques, les données ont été collectées à partir d'un guide d'entretien et sur les réseaux sociaux. Leur analyse a permis d'identifier quatre causes principales. Il s'agit :

1. Du non-respect par les partis du principe d'inclusivité dans la prise de décision pour la mise en place des coalitions. En effet, la démarche de prise de décision qui doit obéir au schéma inversé de la pyramide c'està-dire la consultation préalable de la base et la prise en compte de son avis dans le projet de coalition, n'est pas respectée.

2. Du leadership trop affirmé des leaders des coalitions comme le cas de la LMP. Il est généralement difficile de remplacer un tel leader qui fasse l'unanimité et représente le symbole de l'unité de la coalition.

3. De l'inexistence d'un projet commun clairement formalisé dès le départ entre les partis coalisés. Les coalitions, dès le départ, naissent juste pour la conquête du pouvoir, sans poser les bases d'une durabilité de l'alliance. Une fois l'objectif d'accession au pouvoir exécutif atteint, naissent d'autres enjeux liés à la gestion commune du pouvoir ou liés aux opportunités que présentent les futures échéances électorales. C'est un truisme dans les milieux politiques que la conquête la gestion et la conservation du pouvoir doivent être le but final de toute lutte politique. Par conséquent, elle doit être précédée notamment pour les coalitions qui se mettent en place d'un consensus autour d'un projet de société savamment élaboré et formalisé, indicateur des engagements de chaque membre de la coalition.

4. Du comportement nombriliste des partis politiques membres de la coalition. Les partis membres nourrissent des projets personnels inavoués et, face aux opportunités de positionnement politique remettent en cause les accords conclus dans le cadre de la coalition.

Une coalition est caractérisée par le pluralisme en termes d'idéologies, de valeurs, de représentation géographique. Pour la stabilité et sa durabilité, l'impératif du consensus et du compromis à travers un dialogue permanent s'impose aux animateurs politiques qui la composent. Aussi, la coalition doit dès le départ, sur une base consensuelle et le respect du principe d'inclusivité, s'inscrire dans une démarche de formalisation des accords conclus dans le cadre de la coalition.

Trente ans après l'instauration du multipartisme, les coalitions n'ont pu apporter le changement démocratique recherché. Le changement social en tant que processus, obéit à la logique évolutionniste sociale et sociétale, et 
revêt un caractère révolutionnaire et idéologique, qui jusque là n'est pas observable dans l'arène politique. On assiste toujours à la même logique d'action des coalitions orientée uniquement vers l'éviction du régime au pouvoir qui représente non pas l'adversaire mais l'ennemi politique commun. Elles forment de simples regroupements qui ne recherchent que la majorité électorale, sans une idéologie commune, ni un projet de société novateur clairement élaboré.

La stratégie coalitionnelle n'a pu permettre l'alternance pacifique et le renforcement démocratique par des reformes institutionnelles profondes. Bien au contraire, elle a contribué à la détérioration du l'environnement sociopolitique, l'instabilité des institutions électorales (CEI ; Conseil Constitutionnel etc) au point que le pays est encore en transition démocratique. Chaque cycle électoral est source de tensions et de violences politiques à cause du manque de confiance de l'opposition politique aux institutions chargées des élections.

Au regard de cet échec évident à réaliser le changement politique, il est nécessaire de repenser le modèle de démocratie pour la Côte d'Ivoire en vue de la réalisation d'un renouveau démocratique. Pour ce faire, il faut s'appuyer sur la société civile qui nous semble-t-il, représente une alternative pour booster le changement démocratique tant recherché. Sa contribution significative durant la décennie 2010-2020 à la reforme des institutions électorales et la crédibilité des élections présidentielles respectivement de 2015 et 2020, illustre bien son rôle d'acteur stratégique indispensable dans la construction d'un Etat démocratique. L'exemple de l'APDH (Action pour la Protection des Droits de l'Homme) pour la réforme de la CEI (Commission Electorale Indépendante), et celui de la POECI (Plateforme des Organisations de la société civile pour l'observation des élections en Côte d'Ivoire) et d'INDIGO (Initiative de Dialogue et de Recherche-Action pour la Paix) pour la crédibilité des élections présidentielles respectivement de 2015 et 2020, montrent bien la capacité de la société à contribuer à la transparence et l'inclusivité du processus électoral ainsi qu'à la crédibilité de l'élection et des institutions électorales.

\section{References:}

1. Affi. N. (2018), "Discours d'Affi N'guessan en visite chez Henri Konan Bédié, Président du PDCI-RDA », https://www.youtube.com/watch?v=zS5v2xKgGKM, 15 Mai 2020

2. Akindes. F; Fofana. M; Koné. G. (2010), "Côte d'Ivoire : insurrection et contre-insurrection ", Alternatives sud, Vol.17.

3. Alassane. D. O. (2018), « Discours à l'assemblée générale constitutive du RHDP-le 16 juillet 2018 », https://www.youtube.com/watch?v=fRnn7pJrQt0, 02 Mai 2020 
4. Babo. A. (2008), "Faillite de l'Etat et administration de l'espace public politique par les jeunes en Côte d'Ivoire », CODESRIA,

5. Babo. A. (2011), "Les enjeux de la violence politique chez les milices des jeunes patriotes d'Abidjan en Côte d'Ivoire », ann.univ.de Lomé, sér.lett, tome XXXI-1

6. Bastart. H. (2014) «Constitutions et transitions démocratiques en Côte d'Ivoire de 1990 à 2012 », www.thèses.ulaval.ca, 20 Janvier 2015

7. Bédié. H.K. (2017), «Dans l'actualité /Appel de Daoukro 3: Le Président Bédié appelle à l'union et au respect des engagements", https://www.youtube.com/, 17 Mars 2020

8. Bédié. H.K. (2018), "Communication à son domicile à Daoukro sur le sens de l'appel de Daoukro », https://www.youtube.com/watch?v=zQ85-tmywkA, 19 avril 2019

9. Bitogo, A, 2020, «entretien sur la RFI», 20 juillet 2020

10. Boudon. R ; Besnard. P; Cherkaoui. M ; Lecuyer. B. P. (2012), "Dictionnaire de la sociologie»

11. Bouquet. C. (2005), "Géopolitique de la Côte d'Ivoire. Le désespoir de Kourouma ». Collection Perspectives géopolitiques. Paris, Armand Colin. $315 \mathrm{p}$

12. Ciss. G; Nguini. M. E. O; Stefanoni. P; Talla .J. B. (2013), «L'impératif des alliances en démocratie», Presses Universitaires d'Afrique.

13. Cissé. B. I. (2017), «Meeting de remobilisation des militants RDR à Adjamé », https://www.youtube.com/watch?v=LoIDpUnH8OI, Consulté le 03 mai 2020

14. Commission Electorale Indépendante. (2018), «Résultats des élections municipales et régionales du 13 octobre $2018 »$, https://www.cei-ci.org, Consulté le 13 Mai 2020

15. Dantier. B. (2008), "outils de l'enquête sociologique et enquête sur les outils sociologiques : Georges Granai, techniques de l'enquête sociologique ", extrait de Georges Granai, technique de l'enquête sociologique. Georges Gurvitch, traité de sociologie, tome i. paris, puf

16. Diarassouba. I. (2016), "Usages et signification de la violence syndicale estudiantine dans les universités publiques de Côte d'Ivoire de 1990 à 2010 », Thèse de doctorat en Sociologie, Université Alassane Ouattara, Département d'Anthropologie et de Sociologie

17. Duverger. M. (2003), «Sociologie des partis politiques », in traité de sociologie tome II

18. Duverger. M. (1954), «Les Partis Politiques », Chapitre 7, Armand Colin, 
19. Duverger. M. (1968), « Sociologie des partis politiques », in Traité de sociologie, chapitre 2

20. Gobille. B. (2009), «Comment la stabilité politique se défait-elle? La fabrique de la dépacification en Côte d'Ivoire, 1990-2000 ». Congrès AFSP, Section thématique, $\mathrm{N}^{\circ} 44$,

21. Grand'Maison. J. (1970), "L'église et les idéologies au Québec, Nationalisme et religion ", Volume II: Religion et idéologies politiques, Collection: Pensée actuelle.

22. Hervé. M. (2016), "chronique bibliographique : une nouvelle sociologie des partis politiques en afrique? lectures critiques", Politique africaine $\mathrm{n}^{\circ} 144$, édition Karthala

23. Haeringer. G. (2003), «Sur la coopération dans les jeux non coopératifs», Revue d'économie industrielle, 103 (1)

24. Hyden. G. (1992), «Gouvernance et étude de la politique : Gouverner l'Afrique, vers un partage des rôles ", Colorado, Nouveaux Horizons

25. Institut National de la Statistique. (2015), «recensement général de l'habitat et de la population de 2014: rapport d'exécution et présentation des principaux résultats », http://www.ins.ci/n/documents/RGPH2014_expo_dg.pdf

26. Issiaka K. S. (2017), "Les partis politiques de l'opposition en Afrique : La quête du pouvoir », Presse de l'Université de Montréal

27. Jeune afrique. (2010), "Gbagbo investi candidat pour l'élection présidentielle »,

https://www.jeuneafrique.com/154878/politique/gbagbo-investicandidat-pour-l-lection-pr-sidentielle/, Consulté le 25 Avril 2020

28. Kaudjhis. F.O. (2001) «Les Elections Ivoiriennes de l'An $2000 »$, Journal of African Elections, Vol 1, $\mathrm{N}^{\circ} 1$

29. Kouadio. K.B. (2017), «Invité dans instant de vérité » de l'émission matin bonheur sur la RTI, animée par Gabrielle Zani: https://www.youtube.com/watch?v=ComR-22o6p8, Consulté le 02 Septembre 2020

30. Le Bretony $(\mathrm{M})$; Van der Straeten $(\mathrm{K})$; «Alliances électorales et gouvernementales : la contribution de la théorie des jeux coopératifs à la science politique », Revue d'économie politique Vol. 127,

31. Meier. O. (2019), "Max Weber et la légitimité du pouvoir», https://www.rse-magazine.com/Max-Weber-et-la-legitimite-dupouvoir_a3424.html, Consulté le 16 Mai 2020

32. Mucchielli. R. (2006), "L'analyse du contenu des documents et des communications ». esf,

33. Rocher. G. (2011). «Introduction à la sociologie générale ». Edition, Hurtubise, 
34. Rocher. G. (1968). «Talcott Parsons et la sociologie américaine». Paris, PUF,

35. Toikeusse. M. A. (2019), "Investiture du candidat du RHDP Alassane Ouattara le 26 janvier 2019 », journal télévisé de 20h sur la RTI, www.youtube.com/Watch?v=yvxgaA5bMJ4, Consulté le 05 Septembre 2020

36. Wikipedia. (2000), "Référendum constitutionnel de 2000 », https://fr.wikipédia.org/wiki/R\%/C3\%/A9f\%/, Consulté le 16 Mai 2020

37. Zié, D, C, 2015, "Interview sur la télévision numérique Eventnewtv, à l'émission "en toute vérité » », https://www.youtube.com/watch?, Consulté le 05 Septembre 2020 